\title{
Starobinksy inflation, gravitational contact terms, and the induced Brout-Englert-Higgs boson mass
}

\author{
Christopher T. Hillø* \\ Fermi National Accelerator Laboratory P.O. Box 500, Batavia, Illinois 60510, USA \\ Graham G. Ross $\oplus^{\dagger}$ \\ Rudolf Peierls Centre for Theoretical Physics, University of Oxford, \\ 1 Keble Road, Oxford OX1 3NP, United Kingdom
}

(Received 12 March 2021; accepted 3 June 2021; published 1 July 2021)

\begin{abstract}
The Starobinsky model of inflation remains consistent with observation, forty years after its introduction. It provides a well motivated origin for the scalar inflaton, the "scaleron" with a mass of $O\left(10^{13}\right) \mathrm{GeV}$, emerging as a graviton degree of freedom from $R^{2}$ corrections to Einstein-Hilbert gravity. However the coupling of such a heavy state to the BEH ("Higgs") scalar is problematic as its quantum loop corrections can induce an unacceptably large contribution to the radiatively induced BEH scalar mass. The calculation of these corrections is normally done by Weyl transforming to the Einstein frame, yet at the quantum level Weyl transformations are fraught with ambiguities. However the recent realization that there exist "gravitational contact interactions" largely sidesteps these ambiguities. Such contact terms are necessarily present, coming from t-channel graviton exchange interactions, and they show that the theory defined in the "Jordan frame" is identical to the theory in the Einstein frame, with additional Planck-scale suppressed interactions that take on the form of a Weyl transformation. This avoids ambiguous nonlinear field redefinitions, and reliable loop calculations are possible leading to a consistent low energy theory in an expansion in $1 / M^{2}$. Taking account of the contact terms we study the radiative corrections to the BEH mass in the mixed Higgs $/ R^{2}$ model with explicit scale breaking, and in an extension of the model in which exact scale symmetry is spontaneously broken.
\end{abstract}

DOI: 10.1103/PhysRevD.104.025002

\section{INTRODUCTION}

There has been considerable interest in recent years in the fundamental role of scale symmetry with gravity and the cosmological evolution of the early universe. Scale, or equivalently Weyl invariance (the natural setting for scale symmetry when gravity is incorporated), offers a class of models in which cosmic inflation, the origin of the Planck mass as a dynamical scale symmetry breaking, and potentially large hierarchies seen in nature, can arise as a unified phenomenon.

In the present paper we focus on a leading candidate quantum field theory of inflation known as the Starobinsky Model [1] both in its original form with explicit scale breaking, and in an extension of the model in which exact scale symmetry is spontaneously broken. This is an $R^{2}$ theory,

\footnotetext{
*hill@fnal.gov

'g.ross1@ physics.ox.ac.uk
}

Published by the American Physical Society under the terms of the Creative Commons Attribution 4.0 International license. Further distribution of this work must maintain attribution to the author(s) and the published article's title, journal citation, and DOI. Funded by SCOAP ${ }^{3}$. and as such has an extra physical scalar graviton degree of freedom beyond the two propagating degrees of freedom in the Einstein-Hilbert theory. One can immediately "factorize" the $R^{2}$ interaction, replacing it with a scalar auxiliary field Lagrangian, $\sim \eta^{2} R-\xi \eta^{4}$, and $\eta$ will subsequently acquire kinetic terms. This new field, $\eta$, is known as the "scaleron," emerges automatically in the model and can serve as the inflaton. This in turn leads to a phenomenologically successful inflationary model provided the scaleron is sufficiently heavy of $O\left(10^{13}\right) \mathrm{GeV}$. We are interested in the effective quantum field theory aspects of this scheme. In particular we explore the naturalness of the light Brout-Englert-Higgs (BEH, "Higgs") scalar [2-4] of the Standard Model when it is introduced into the theory and interacts with the scaleron state, the "Higgs $/ R^{2}$ " model. This is particularly interesting in the scale invariant version of the model because scale invariance forbids a bare BEH mass and it is only generated on spontaneous breaking of the symmetry.

A standard technology used in performing loop calculations in effective field theory in these schemes involves the "Weyl transformation." While an exact transformation classically, the Weyl transformation involves nonlinear field redefinitions, and leads to a morass of ambiguities 
at the quantum level. Mainly, Weyl transformations can transform a classical theory with nonminimal couplings of scalars to gravity, as $F(\phi) R$, into any other coupling, $C(\phi) R$. There is a special frame, $C(\phi)=M^{2}$, called the "Einstein frame," where the nonminimal couplings are absent. However, one would think any frame should be as good as any other and indeed some authors invoke special properties of other frames, such as those with "conformal coupling," $-\frac{1}{12} \phi^{2} R$.

Recently, however, we pointed out that if one considers the $t$-channel exchange of gravitons in a theory with Planck scale and nonminimal interactions, $\left(M^{2}+F(\phi)\right) R$, a novel phenomenon occurs [5]: the theory has contact term interactions. In weak field approximation, $g_{\mu \nu} \approx \eta_{\mu \nu}+h_{\mu \nu} / M$ the scalar curvature has a leading $F(\phi) \partial^{2} h$ term. ${ }^{1}$ In a Feynman diagram this implies a vertex proportional to $q^{2}$, which in turn cancels the $1 / q^{2}$ in the graviton propagator. This means that the would-be long range graviton exchange potential arising from the nonminimal terms collapses to a deltafunction, and becomes a set of local operators. The contact term integrates out the $F(\phi) R$ term and replaces it with higher dimension operators, $\sim F D^{2} F / M^{2}$ and $\sim F T_{\mu}^{\mu} / M$.

In exploring radiative corrections we find, if we do not take the contact term into account, the resulting induced BEH scalar mass is dependent upon the frame choice. Effectively the different Weyl frames, as conventionally treated in the absence of the contact term, are then different theories. Therefore, it would appear that Weyl transformations are incompatible with quantum theory. Indeed, this incompatibility has been argued to be the case long ago by M. Duff [6]. This issue has been addressed by [7], who find that the frame dependence is remedied by working on-mass shell for background fields, i.e., by imposing equations of motion. But what of off-shell quantities, such as $\beta$-functions? How is uniqueness of the theory realized as an action?

It is important to realize that the contact term, in the $1 / M_{P}^{2}$ expansion, induces the higher dimension local operators that are part of the effective action. In effect, the contact term induced operators are hidden parts of the full effective action in any given frame. In any putative "Jordan frame" the nonminimal interaction will always be replaced by these new operators. The contact term in gravity, though it involves no field redefinitions, has the same structure in any frame as a Weyl transformation leading to the Einstein frame. ${ }^{2}$ However, it is important to note that the contact term is not a Weyl transformation.

\footnotetext{
${ }^{1}$ This term is projected to zero in the Einstein-Hilbert action where $\int d^{4} x \sqrt{-g} M^{2} D^{2} h$ is a total divergence

${ }^{2}$ In Ref. [5] the leading behavior in $1 / M^{2}$ was derived, but presumably the t-channel diagrams can be resummed to yield the full Weyl transformation form. While we have not explicitly proved this, one can see evidence of it by considering multigraviton exchange diagrams, or considering iteration of the Einstein equation for $R$.
}

Due to the contact terms we see that the Jordan frame, which has anomalous couplings of the scalars to the Ricci scalar, is identical to the Einstein frame with only an Einstein Hilbert term, $M_{P}^{2} R$, and higher dimension operators. The contact terms can be seen as arising by judiciously using the equation of motion for $R$ within the nonminimal terms, hence it is not surprising that the consistency among frames observed in [7] occurs on-shell. There the imposition of the equation of motion implicitly implements the contact terms. However, the contact term assures that the $\beta$-functions computed in the Einstein frame are the unique and correct ones.

Contact terms are familiar elsewhere in physics. A wellknown example occurs in electroweak phsyics, where we have "penguin diagram," in which electroweak vertex corrections to gluon exchange generates contact terms. These lead to new local 4-fermion operators which mediate processes such as $K \rightarrow 2 \pi$. The contact term is classical, of $\mathcal{O}\left(\hbar^{0}\right)$ arising from tree diagrams, yet contact terms are an exception to the rule that corrections to the action come only from single particle irreducible (loop) diagrams. The contact term is an $a b$ initio hidden yet essential part of the physics. Moreover, one would be double counting to add the contact terms to the action without removing the $q^{2}$ vertices as well.

One still has an ambiguity of choice of source currents in an effective potential calculation in the Einstein frame an example is given in Appendix C. This is a "user option," analogous to whether one computes a magnet's potential as function $\vec{M}$ (magnetization) or $\vec{M}^{2}$. Many choices are possible, and lead to physically different potentials and interpretations. Presently we are interested in the order parameter that can serve optimally as the inflaton. In studying inflation it is necessary to consider the evolution of fields away from equilibrium and motivates one to choose a variable that diagonalizes the kinetic term in the absence of a constant background field. For the original Starobinsky model this leads one to a natural fundamental variable eliminating the remaining ambiguity.

In calculating the effective action in the ColemanWeinberg method [8], it is important to realize that the source terms lead to a result with "on-shell" classical background fields. Merely shifting and computing without sources can lead to results that are dependent on the choice quantum fluctuations (the actual field we integrate in the path integral). For potentials, "on-shell" means that static classical background fields are VEVs. Since we are interested in the effective potential far from the true potential minimum we add the source terms that shift these fields to arbitrary classical values. These shifted fields are then minima of the full potential with sources, where the currents have deformed the potential. In the special case that background fields are in the true local minimum of the classical potential, where the sources vanish, then the fields are automatically on-shell. With a given set of sources and 
on-shell background fields, the effective potential will then be invariant under different choices of the quantum fluctuation fields. A proof and discussion of this is given in Appendix B.

We also introduce an alternative method to compute the effective potential using the renormalization group (RG). This is an expeditious method based on the fact that the rather complicated and nonrenormalizable form of the potential at short-distance (Planck-scale) contains a subset of "relevant operators" that propagate logarithmically into the infrared and define the low energy theory. We write down a generic set of relevant operators, match their coefficients at $M$ to the Starobinsky potential, and compute the evolution of their couplings into the infrared with conventional RG equations. This is similar to a second order phase transition in condensed matter physics, where there is approximate scale invariance and sensitivity to the complicated short-distance physics is erased, leading to universal low energy results. Presently, the low energy form of the Higgs mass is found to be identical to the ColemanWeinberg form and can be directly connected to the trace anomaly [9]. This is a powerful and efficient technique, and will be developed further elsewhere [10].

Contact terms thus mandate a unique and well defined formulation of the theory in the Einstein frame. In what follows we will apply this to focus upon two settings for the Starobinsky model. We will begin with a nonscale-invariant theory in which the Planck mass is present as a fundamental scale and the model is extended to include the BEH scalar. We will also analyse a Weyl invariant version of the Starobinsky model including the BEH scalar in which the fundamental mass scales arise by spontaneous symmetry breaking [11]. This requires an additional scalar field and forms the basis of the modern Weyl invariant field theory approach [12-26].

Here the spontaneous breaking of the Weyl-scale symmetry is a consequence of the conserved Weyl current, $K_{\mu}=\partial_{\mu} K$ where $K$ is a scalar function of the scalar fields. Any conserved current will redshift to zero in a general expansion of the universe, and so too the Weyl current. However, since the Weyl current is a derivative of a scalar $K$, we see that $K$ will therefore redshift to an arbitrary constant, $\bar{K}$. This is the order parameter of the spontaneous symmetry breaking, and when it acquires a nonzero VEV the symmetry is broken. We call this "inertial symmetry breaking" [27] because it does not involve a potential. There will be a dilaton, and the decay constant of the dilaton is $\sqrt{2 \bar{K}}$, and is also proportional to the Planck mass.

\section{THE MIXED Higgs $/ R^{2}$ MODEL}

\section{A. The action}

We focus presently upon the mixed Higgs $/ R^{2}$ model that extends the Starobinsky inflationary model [1] to include the BEH boson. The model has the action:

$$
\begin{aligned}
S= & \int \sqrt{-g}\left(\frac{1}{2} g^{\mu \nu} \partial_{\mu} H \partial_{\nu} H-\frac{1}{6 f_{0}^{2}} R^{2}\right. \\
& \left.-\frac{1}{12} \alpha_{H} H^{2} R+\frac{1}{2} M^{2} R-V(H)\right)
\end{aligned}
$$

where $M$ is the Planck mass and $R(g)$ is the Ricci scalar of the metric $g_{\alpha \beta}$. Here we also include the Standard Model $\mathrm{BEH}$ scalar isodoublet $\mathcal{H}$, which, for convenience we will treat $\mathcal{H}$, as a real scalar field $H$. This can be identified with the physical BEH isodoublet scalar of the Standard Model when $\mathcal{H}$ is written in the unitary gauge $\mathcal{H}=(0, H / \sqrt{2})$ and we assume the electroweak gauge fields are pure-gauge configurations. Then $D_{\mu} \mathcal{H} \rightarrow \partial_{\mu} H$ and $\mathcal{H}^{\dagger} \mathcal{H} \rightarrow \frac{1}{2} H^{2}$. We have allowed for a nonminimal coupling of $H$ proportional to $\alpha_{H}$. Even if this is initially set to zero it is generated radiatively by Standard Model couplings [28,29]. We will largely ignore $V(H)$ as it plays a subdominant role in the radiative corrections.

Since the $R^{2}$ term involves fourth order derivatives it contains an additional (scalar) degree of freedom [30,31]. To make this explicit it is conventional to reduce the fourth order derivatives to second order by introducing a static auxiliary field, $\eta$, with the action now given by:

$$
\begin{aligned}
S= & \int \sqrt{-g}\left(\frac{1}{2} g^{\mu v} \partial_{\mu} H \partial_{\nu} H-\frac{1}{12} \alpha_{\eta} \eta^{2} R\right. \\
& \left.-\frac{1}{12} \alpha_{H} H^{2} R+\frac{1}{2} M^{2} R-\frac{\xi^{\prime}}{4} \eta^{4}\right)
\end{aligned}
$$

where the equation of motion yields:

$$
\eta^{2}=-\frac{1}{6} \frac{\alpha_{\eta}}{\xi^{\prime}} R \quad \text { and }: \quad f_{0}^{2}=\frac{24 \xi^{\prime}}{\alpha_{\eta}^{2}} .
$$

The coupling $\xi^{\prime}$ and the value of $\alpha_{\eta}$ are relative. We can define a new $\xi=\xi^{\prime} / \alpha_{\eta}^{2}$ and rescale $\eta^{2}$ so that $\alpha_{\eta}=1$ and,

$$
-\frac{1}{12} \alpha_{\eta} \eta^{2} R-\frac{\xi^{\prime}}{4} \eta^{4} \rightarrow-\frac{1}{12} \eta^{2} R-\frac{\xi}{4} \eta^{4} .
$$

We will take $\alpha_{\eta}^{2}=1$ to be "standard normalization," in which case we have $f_{0}^{2}=24 \xi$. In the standard normalization the action becomes:

$$
S=\int \sqrt{-g}\left(\frac{1}{2} g^{\mu v} \partial_{\mu} H \partial_{\nu} H+\frac{1}{2} M^{2} R \Omega^{2}-\frac{\xi}{4} \eta^{4}\right)
$$

where we define:

$$
\Omega^{2}=1-\frac{\alpha_{H}}{6 M^{2}} H^{2}-\frac{1}{6 M^{2}} \eta^{2} \equiv \exp \left(\frac{2 \chi}{\sqrt{6} M}\right)
$$

and $\chi$ will play the role of the inflaton. 
While this formulation is done in the "Jordan frame," as shown in [5], contact interactions generated by tree level graviton exchange significantly modify the action. In this sense the Jordan frame does not really exist, as it is driven to the Einstein frame by the contact terms. In practice, rather than carrying out the laborious process of calculating the contact terms, we can formally perform a Weyl transformation to go to an Einstein-Hilbert action:

$$
\begin{array}{rlrl}
g_{\mu \nu}(x) & \rightarrow \Omega^{-2} g_{\mu \nu}(x) & g^{\mu \nu}(x) & \rightarrow \Omega^{2} g^{\mu \nu}(x) \\
\sqrt{-g} & \rightarrow \sqrt{-g} \Omega^{-4} & R\left(\Omega^{-2} g\right)=\Omega^{2} R(g)+6 \Omega^{3} D \partial \Omega^{-1} .
\end{array}
$$

We emphasize however that the contact terms are not a Weyl transformation and preserve the original metric, though formally the resulting structure of the theory is that of the Weyl transformation.

Then we have from Eq. (6),

$$
\eta^{2}=6 M^{2}\left(\exp \left(\frac{2 \chi}{\sqrt{6} M}\right)-\left(1-\frac{\alpha_{H}}{6 M^{2}} H^{2}\right)\right),
$$

and the potential takes the form:

$$
\frac{\xi}{4} \Omega^{-4} \eta^{4}=\frac{3}{8} M^{4} f_{0}^{2}\left(1-\exp \left(-\frac{2 \chi}{\sqrt{6} M}\right)\left(1-\frac{\alpha_{H}}{6 M^{2}} H^{2}\right)\right)^{2} .
$$

We now turn to two different treatments of the effective potential. One follows the Coleman-Weinberg approach, introducing sources and performing an expansion to order $\hbar$. The second approach uses the renormalization group and is much simpler.

\section{B. A Coleman-Weinberg calculation of the effective potential}

The Coleman-Weinberg potential [8] results from a WKB approximation to computing a Gaussian integral in field theory. We add source terms to dynamically shift the classical values of the fields to nonzero on-shell VEVs. Presently, we choose a source term $J \sqrt{6} M \ln \Omega+K H$. The parametrization of the fields is then arbitrary and we will assume, $\chi=\sqrt{6} M \ln \Omega$ and $H$, and then note that $3 M^{2} \partial \ln \Omega \partial \ln \Omega=\frac{1}{2} \partial_{\rho} \chi \partial^{\rho} \chi$. The Einstein frame action is then:

$$
\begin{aligned}
S= & \int \sqrt{-g}\left(\frac{1}{2} \Omega^{-2} \partial_{\rho} H \partial^{\rho} H+\frac{1}{2} \partial_{\rho} \chi \partial^{\rho} \chi+\frac{1}{2} M^{2} R\right. \\
& \left.-\frac{\xi}{4} \Omega^{-4} \eta^{4}+J \sqrt{6} M \ln \Omega+K H\right) .
\end{aligned}
$$

We emphasize that Eq. (10) is a result of the contact terms, and the metric appearing is the original metric of Eq. (2).

We now expand in in $\sqrt{\hbar} \equiv \epsilon$ :

$$
H=h+\epsilon x, \quad \chi=\kappa+\epsilon y, \quad \sqrt{\hbar}=\epsilon
$$

where $(h, \kappa)$ are classical background fields and $(x, y)$ are quantum fluctuation scalar fields, integrated in the path integral. We also introduce the following useful functions of the classical background fields:

$$
P=\exp \left(-\frac{\kappa}{\sqrt{6} M}\right), \quad p=\sqrt{\frac{\alpha_{H}}{6 M^{2}}} h, \quad q=\sqrt{\frac{1}{6 M^{2}}} h .
$$

The action to $\mathrm{O}(\hbar)=\epsilon^{2}$ becomes:

$$
\begin{aligned}
S= & \int \sqrt{-g}\left(\frac{1}{2} M^{2} R+\frac{1}{2} \epsilon^{2} P^{2} \partial_{\rho} x \partial^{\rho} x\right. \\
& \left.+\frac{1}{2} \epsilon^{2} \partial_{\rho} y \partial^{\rho} y-V(J, K)\right),
\end{aligned}
$$

where the potential of Eq. (9) including sources is given by:

$$
V(J, K)=\frac{\xi}{4} \Omega^{-4} \eta^{4}+J(\kappa+\epsilon y)+K(h+\epsilon x),
$$

where,

$$
\begin{aligned}
\frac{\xi}{4} \Omega^{-4} \eta^{4} \\
=\frac{3}{8} M^{4} f_{0}^{2}\left(1-P^{2}\left(1-\frac{2}{\sqrt{6} M} y \epsilon+\frac{1}{3 M^{2}} y^{2} \epsilon^{2}+O\left(\epsilon^{3}\right)\right)\right. \\
\left.\quad \times\left(1-\frac{\alpha_{H}}{6 M^{2}}\left(h^{2}+2 h \epsilon x+\epsilon^{2} x^{2}\right)\right)\right)^{2}
\end{aligned}
$$

The sources are determined by the condition that the potential $V(J, K)$ be evaluated at the minimum, whence the linear terms in $\epsilon$ vanish. Since the particular sources we have chosen couple linearly to the fields they do not affect the quadratic term, $O\left(\epsilon^{2}\right)$, in the expansion of the potential (see Appendix B for an exception). Then performing the Legendre transformation:

$V(\kappa, h)=V(J, K)-J \frac{d}{d J} V(J, K)-K \frac{d}{d J} V(J, K)$

with $C=\frac{3}{8} M^{4} f_{0}^{2}=9 M^{4} \xi$, we expand $V(\kappa, h)$ to $O\left(\epsilon^{2}\right)$ to obtain the potential, $V(\kappa, h)$, 
$V(\kappa, h)=V_{\text {classical }}+\frac{C}{M^{2}} \epsilon^{2}\left(\frac{1}{3}\left(1-P^{2}\left(1-p^{2}\right)\right) P^{2}\left(\alpha_{H} x^{2}-4 \sqrt{\alpha_{H}} p x y-2 y^{2}\left(1-p^{2}\right)\right)+\frac{2}{3} P^{4}\left(\sqrt{\alpha_{H}} p x+y\left(1-p^{2}\right)\right)\right)^{2}$

where the classical potential is $V_{\text {classical }}=C\left(1-P^{2}\left(1-p^{2}\right)\right)^{2}$.

\section{One loop radiative corrections}

At $O(\hbar)$, the quadratic term in the fluctuations can be written as an unrenormalized mass squared matrix among the quantum fluctuations $(x, y)$ :

$$
\frac{1}{2}\left(\begin{array}{l}
y \\
x
\end{array}\right)^{T} \Xi_{0}^{2}\left(\begin{array}{l}
y \\
x
\end{array}\right) \quad \text { where, } \quad \Xi_{0}^{2}=\frac{2 C}{M^{2}}\left(\begin{array}{cc}
\frac{2}{3} P^{2}\left(p^{2}-1\right)\left(1-2 P^{2}\left(1-p^{2}\right)\right) & -\frac{2}{3} P^{2} \sqrt{\alpha_{H}} p\left(1-2 P^{2}\left(1-p^{2}\right)\right) \\
-\frac{2}{3} P^{2} \sqrt{\alpha_{H}} p\left(1-2 P^{2}\left(1-p^{2}\right)\right) & \frac{1}{3} P^{2} \alpha_{H}\left(1-P^{2}+3 P^{2} p^{2}\right)
\end{array}\right) .
$$

Likewise the kinetic terms for $(x, y)$ can be written:

$$
\frac{1}{2} \epsilon^{2} P^{2} \partial_{\rho} x \partial^{\rho} x+\frac{1}{2} \epsilon^{2} \partial_{\rho} y \partial^{\rho} y=\left(\begin{array}{c}
\partial y \\
\partial x
\end{array}\right)^{T} Z\left(\begin{array}{c}
\partial y \\
\partial x
\end{array}\right)
$$

where,

$$
Z=\left(\begin{array}{cc}
1 & 0 \\
0 & P^{2}
\end{array}\right) \quad \sqrt{Z}^{-1}=\left(\begin{array}{cc}
1 & 0 \\
0 & P^{-1}
\end{array}\right)
$$

The physical, renormalized mass squared matrix is therefore:

$$
\Xi^{2}=\sqrt{Z}^{-1} \Xi_{0}^{2} \sqrt{Z^{-1}}=\frac{8}{3} \frac{C}{M^{2}}\left(\begin{array}{cc}
-\frac{1}{2} P^{2}\left(1-p^{2}\right)\left(1-2 P^{2}\left(1-p^{2}\right)\right. & -\sqrt{\alpha_{H}} p P\left(\frac{1}{2}-P^{2}\left(1-p^{2}\right)\right) \\
-\sqrt{\alpha_{H}} p P\left(\frac{1}{2}-P^{2}\left(1-p^{2}\right)\right) & \frac{1}{4} \alpha_{H}\left(1-P^{2}\left(1-3 p^{2}\right)\right) .
\end{array}\right)
$$

Strictly following the analysis of Coleman and Weinberg [8] we should construct the eigenvalues of the mass squared matrix, denoted as $\left(\Xi_{1}^{2}, \Xi_{2}^{2}\right)$. The potential then takes the form, subject to renormalization conditions,

$$
\Gamma=-\frac{1}{64 \pi^{2}} \Xi_{1}^{4} \ln \left(M^{2} / \Xi_{1}^{2}\right)-\frac{1}{64 \pi^{2}} \Xi_{2}^{4} \ln \left(M^{2} / \Xi_{2}^{2}\right)
$$

(see Appendix A). Here the mass eigenstates correspond to the scaleron and the BEH scalar. Given the large mass of the scaleron it dominates the contribution to Eq. (22) and to an excellent approximation we can can define the resulting effective potential as:

$$
\Gamma=-\frac{1}{64 \pi^{2}} \operatorname{Tr}\left(\Xi^{4}\right)\left(\ln \left(M^{2} / m^{2}\right)\right)
$$

where $m$ is the scaleron mass and we have:

$$
\operatorname{Tr}\left(\Xi^{4}\right)=\frac{16}{9} \frac{C^{2}}{M^{4}}\left(P^{4}\left(1-p^{2}\right)^{2}\left(1-2 P^{2}\left(1-p^{2}\right)\right)^{2}+8 \alpha_{H} p^{2} P^{2}\left(\frac{1}{2}-P^{2}\left(1-p^{2}\right)\right)^{2}+\frac{1}{4} \alpha_{H}^{2}\left(1-P^{2}\left(1-3 p^{2}\right)\right)^{2}\right)
$$

This is the leading order result in $\hbar=\epsilon^{2}$ valid to all orders in $(\kappa, h)$. We are interested in calculating the correction to the $\mathrm{BEH}$ mass at the end of inflation in the limit of small $\kappa \rightarrow 0$, hence $P=\exp \left(-\frac{\kappa}{\sqrt{6}}\right) \rightarrow 1$ and

$$
\operatorname{Tr}\left(\Xi^{4}\right) \rightarrow \frac{16}{9} \frac{C^{2}}{M^{4}}\left(1+\left(-\frac{\alpha_{H}}{M^{2}}+\frac{1}{3} \frac{\alpha_{H}^{2}}{M^{2}}\right) h^{2}+O\left(h^{4}\right)\right)
$$


and, with $f_{0}^{2}=24 \xi, C=(3 / 8) M^{4} f_{0}^{2}=9 M^{4} \xi$, the loop induced Higgs mass term is then:

$$
\begin{aligned}
\Gamma & =-\frac{3}{4 \pi^{2}} M^{2} \xi^{2} \alpha_{H}\left(\alpha_{H}-3\right) h^{2} \ln \left(M^{2} / m^{2}\right) \\
& =-\frac{1}{768 \pi^{2}} M^{2} f_{0}^{4} \alpha_{H}\left(\alpha_{H}-3\right) h^{2}\left(\ln \left(M^{2} / m^{2}\right)\right)
\end{aligned}
$$

Note the parameters refer to the action of Eq. (1) where $\xi$ is rescaled to standard normalization. This is our key result for the NSI Starobinsky model, and disagrees with previous results, such as [18].

\section{Renormalization group approach}

The effective potential approach used in the preceding section is a well-defined procedure. Its relationship to other fundamental quantities in the field theory, such as the $\beta$-functions and trace anomalies, is not so obvious. However, we can easily obtain the preceding results with the renormalization group equations. The connection to the trace anomaly is then direct, as in Ref. [9].

We construct a generic theory consisting of "relevant operators" up to dimension 4, that participate in the logarithmic running of the relevant coupling constants. We then impose boundary conditions at the UV (Planck) scale that match the parameters of this generic potential to the nonscale invariant Starobinsky model.

Consider the following generic field theory:

$$
S=\int \frac{1}{2} \partial_{\rho} H \partial^{\rho} H+\frac{1}{2} \partial_{\rho} \chi \partial^{\rho} \chi-W(\chi, H)
$$

where the potential is

$$
\begin{aligned}
W(\chi, H)= & \frac{1}{2} \mu_{1}^{2} \chi^{2}+\frac{1}{2} \mu_{2}^{2} H^{2}+m_{1} \chi^{3}+m_{2} \chi H^{2} \\
& +\frac{\lambda_{1}}{4} \chi^{4}+\frac{\lambda_{2}}{4} H^{4}+\frac{\lambda_{3}}{2} H^{2} \chi^{2} .
\end{aligned}
$$

This maintains the symmetry $H \rightarrow-H$. We expand the fields $(\chi, H)$ in VEVs + fluctuations, $(\chi, H) \rightarrow(\kappa+\hbar x, h+\hbar y)$ and integrate the fields $(x, y)$ to obtain one-loop RG equations (this can be done efficiently by the $\operatorname{Tr}\left(\Xi^{4}\right)$ method as in Appendix A). There are then seven renormalization group equations in terms of $(t=\ln (\mu))^{3}$ :

\footnotetext{
${ }^{3}$ We specialize to the case $P=1$ which would otherwise lead to wave-function renormalization corrections. We also presently ignore the interesting issue of $\alpha_{i}$ running which necessitates regeneration of the contact term. These issues will be dealt with in a subsequent paper [10].
}

$$
\begin{aligned}
8 \pi^{2} \frac{\partial}{\partial t} \mu_{2}^{2} & =-\left(3 \lambda_{2} \mu_{2}^{2}+4 m_{2}^{2}+\lambda_{3} \mu_{1}^{2}\right) \\
8 \pi^{2} \frac{\partial}{\partial t} \mu_{1}^{2} & =-\left(2 m_{2}^{2}+\lambda_{3} \mu^{2}+18 m_{1}^{2}+3 \lambda_{1} \mu_{1}^{2}\right) \\
8 \pi^{2} \frac{\partial}{\partial t} m_{1} & =-\left(9 \lambda_{1} m_{1}+\lambda_{3} m_{2}\right) \\
8 \pi^{2} \frac{\partial}{\partial t} m_{2} & =\left(3 \lambda_{2} m_{2}+4 \lambda_{3} m_{2}+3 \lambda_{3} m_{1}\right) \\
8 \pi^{2} \frac{\partial}{\partial t} \lambda_{1} & =\left(9 \lambda_{1}^{2}+\lambda_{3}^{2}\right) \\
8 \pi^{2} \frac{\partial}{\partial t} \lambda_{2} & =\left(9 \lambda_{2}^{2}+\lambda_{3}^{2}\right) \\
8 \pi^{2} \frac{\partial}{\partial t} \lambda_{3} & =\lambda_{3}\left(3 \lambda_{2}+4 \lambda_{3}+3 \lambda_{1}\right) .
\end{aligned}
$$

While these can be formally integrated in leading log approximation, here we are only interested in the single $\log$ solutions, such as,

$$
\lambda_{1}=\lambda_{1_{0}}-\frac{1}{8 \pi^{2}}\left(\lambda_{3_{0}}^{2}+9 \lambda_{1_{0}}^{2}\right) \ln \left(\frac{M}{\mu}\right)
$$

where $\lambda_{i_{0}}$ is the initial value of $\lambda_{i}$ at $\mu=M$ (see Appendix A).

To obtain the Higgs mass we use the NSI potential as the boundary condition on the theory when $\mu=M$. From Eq. (9):

$$
\frac{\xi}{4} \Omega^{-4} \eta^{4}=\frac{3}{8} M^{4} f_{0}^{2}\left(1-\exp \left(-\frac{2 \kappa}{\sqrt{6} M}\right)\left(1-\frac{\alpha_{H}}{6 M^{2}} h^{2}\right)\right)^{2}
$$

Expanding this to quartic order in the fields and matching coefficients, our generic potential takes the form at the Planck-scale:

$$
\begin{aligned}
W(\kappa, h) / f_{0}^{2}= & \frac{1}{4} M^{2} \kappa^{2}-\frac{1}{2 \sqrt{6}} M \kappa^{3}+\frac{\alpha_{H}}{4 \sqrt{6}} M \kappa h^{2} \\
& -\frac{\alpha_{H}}{8} \kappa^{2} h^{2}+\frac{7}{72} \kappa^{4}+\frac{1}{96} \alpha_{H}^{2} h^{4}
\end{aligned}
$$

In particular we see that, at the Planck-scale, $M$, the initial values of the parameters relevant to the Higgs mass are

$$
\begin{aligned}
& \mu_{2_{0}}^{2}=0 \quad m_{2_{0}}=\frac{\alpha_{H_{0}}}{4 \sqrt{6}} M f_{0}^{2} \\
& \mu_{1_{0}}^{2}=\frac{1}{2} M^{2} f_{0}^{2} \quad \lambda_{3_{0}}=-\frac{1}{4} \alpha_{H_{0}} f_{0}^{2}
\end{aligned}
$$

Therefore, combining these results with the first line of Eqs. (29), the Higgs mass is determined at a lower energy scale $\mu=m$ in the single log approximation: 


$$
\mu_{2}^{2}(\mu)=-\frac{1}{384 \pi^{2}} f_{0}^{4} M^{2} \alpha_{H}\left(\alpha_{H}-3\right) \ln \left(M^{2} / m^{2}\right)
$$

yielding the Higgs potential:

$$
\Gamma=\frac{1}{2} \mu_{2}^{2} h^{2}=-\frac{1}{768 \pi^{2}} f_{0}^{4} M^{2} \alpha_{H}\left(\alpha_{H}-3\right) h^{2} \ln \left(M^{2} / m^{2}\right)
$$

in exact agreement with Eq. (26).

We have presented the renormalization group (RG) approach here to illustrate that it is much simpler and potentially more powerful than the effective potential calculation obtained previously. The RG clarifies the ambiguities, which now largely lie in the choice of the field parametrizations in the UV boundary conditions of the theory [9].

Note, moreover, that at this order the running of the Higgs mass does not involve the running of the $\alpha_{i}$. The running of the $\alpha_{i}$ leads to the tricky problem of the regeneration of the contact term, which we will treat elsewhere [10]. However, ultimately, a stable Higgs mass would likely necessitate an infrared fixed point of these RG equations.

\section{THE SCALE INVARIANT MIXED HIGGS/ $\boldsymbol{R}^{2}$ MODEL}

We can readily construct a scale invariant version of the mixed Higgs $/ R^{2}$ model $[32,33]$ in which the inflationary parameters are predicted to be close to the original Starobinsky model which is in good agreement with present observations. As before, to obtain the observed amplitude of the perturbations $f_{0} \sim 10^{-5}$ requires that the scaleron has mass of $O\left(10^{13}\right) \mathrm{GeV}$.

The scale invariant action is

$$
\begin{aligned}
S= & \int \sqrt{-g}\left(\frac{1}{2} g^{\mu v} \partial_{\mu} \phi \partial_{\nu} \phi+\frac{1}{2} g^{\mu v} \partial_{\mu} H \partial_{\nu} H-\frac{1}{12} \alpha_{\phi} \phi^{2} R\right. \\
& \left.-\frac{1}{12} \alpha_{\eta} \eta^{2} R-\frac{1}{12} \alpha_{H} H^{2} R-\frac{\lambda}{4} \phi^{4}-\frac{\xi}{4} \eta^{4}-\frac{\omega}{4} H^{4}\right)
\end{aligned}
$$

Here we again have the auxiliary field $\eta$, which can be integrated out to recover the $R^{2}$ term as in the nonscale invariant model of Sec. II.

Note that, in the limit $\alpha_{\phi}=\alpha_{H}$ and $\lambda=\omega=0$, the theory is $S O(2)$ invariant. In this limit the $\mathrm{BEH}$ scalar is massless and the symmetry is not broken by radiative corrections. As a result the Higgs mass $\beta$-function must vanish in this limit.

Note the action is classically invariant under the Weylscale transformation:

$$
\begin{aligned}
g_{\mu \nu}(x) & =e^{2 \epsilon} g_{\mu \nu}(x) \quad g^{\mu \nu}(x)=e^{-2 \epsilon} g^{\mu \nu}(x) \\
(\phi, \eta, H) & =e^{-\epsilon}(\phi, \eta, H)
\end{aligned}
$$

This leads to a conserved Noether current:

$$
\begin{aligned}
K_{\mu} & =\partial_{\mu} K \quad \text { where } \\
K & =\frac{1}{2}\left(\left(1-\alpha_{\phi}\right) \phi^{2}+\left(1-\alpha_{H}\right) H^{2}-\eta^{2}\right)
\end{aligned}
$$

The current has the special form, in analogy to topological currents, of being a derivative of a scalar, $K$, which we refer to as the "kernal." A pre-Plankian phase will cause redshifting and the conservation of $K_{\mu}$ implies that $K \rightarrow \bar{K}$ constant. This "condensation" of the kernal, $K$, spontaneously breaks scale symmetry and has been dubbed "inertial symmetry breaking," [27], since it makes no reference to a potential. The phase transition is from a highly disordered state to an ordered state and occurs when fluctuations in $K$ become small compared to its average VEV, i.e., $\left|\left\langle K^{2}\right\rangle-\bar{K}^{2}\right| \ll \bar{K}^{2}$.

We can rewrite the original dynamical $K$ as

$$
2 K=\left(\left(1-\alpha_{\phi}\right) \hat{\phi}^{2}+\left(1-\alpha_{H}\right) \hat{H}^{2}-\hat{\eta}^{2}\right) e^{2 \sigma / f}
$$

where $f=\sqrt{2 \bar{K}}$ canonically normalizes $\sigma$ in the broken phase, and the dilaton, $\sigma$, apparently decouples in the Jordan frame [34] but acquires derivative couplings to Standard Model fields via contact terms or, formally, via the Weyl transformation going to the Einstein frame. The inertial symmetry breaking can be viewed as the red shifting of the dilaton to a constant VEV, $\sigma \rightarrow \sigma_{0}$, and thus $K \rightarrow \bar{K}$ (the VEV of the dilaton has no absolute meaning and is only defined relative to the other field VEV's, and in fact the dilaton itself is only well defined in the ordered phase as a Nambu-Goldstone boson when $K \rightarrow \bar{K}$ ).

The resulting constraint is

$$
2 \bar{K}=\left(1-\alpha_{\phi}\right) \hat{\phi}^{2}-\hat{\eta}^{2}+\left(1-\alpha_{H}\right) \hat{H}^{2}=\text { constant. }
$$

In what follows we will determine the radiative corrections to the Higgs mass following from the action Eq. (36). Dropping the dilaton, which does not contribute significantly to the radiative correction, the action becomes:

$$
\begin{aligned}
S= & \int \sqrt{-g}\left(\frac{1}{2} g^{\mu v} \partial_{\mu} \hat{\phi} \partial_{\nu} \hat{\phi}+\frac{1}{2} g^{\mu \nu} \partial_{\mu} \hat{H} \partial_{\nu} \hat{H}\right. \\
& +\frac{1}{2} M^{2} R\left(-\frac{1}{6 M^{2}} \alpha_{\phi} \hat{\phi}^{2}-\frac{1}{6 M^{2}} \hat{\eta}^{2}-\frac{1}{6 M^{2}} \alpha_{H} \hat{H}^{2}\right) \\
& \left.-\frac{\lambda}{4} \hat{\phi}^{4}-\frac{\xi}{4} \hat{\eta}^{4}-\frac{\omega}{4} \hat{H}^{4}\right)
\end{aligned}
$$

In what follows, for notational convenience, all fields are understood to be hatted fields although we do not display 
the hats. The constraint of Eq. (40) means that we can consider any pair of the $(\phi, \eta, H)$ as unconstrained, while the remaining field is then determined by the constraint to be a function of the other two. The case that $\eta$ is eliminated manifestly preserves the underlying $S O(2)$ symmetry where $(\phi, H)$ form a doublet. The case that $\phi$ is eliminated hides this symmetry but gives a calculation very similar to the nonscale invariant case. As a gratifying check on the analysis we have found that both routes lead to the same result for the BEH scalar mass.

\section{A. Calculation manifestly preserving the underlying $S O(2)$ symmetry structure}

Defining $\bar{K}=3 M^{2}$ we presently use the constraint, Eq. (40), to eliminate the $\eta^{2}$ term in the coefficient of the Ricci scalar in the action, Eq. (41). We thus obtain:

$$
\begin{aligned}
S_{A}= & \int \sqrt{-g}\left(\frac{1}{2} g^{\mu v} \partial_{\mu} \phi \partial_{\nu} \phi+\frac{1}{2} g^{\mu v} \partial_{\mu} H \partial_{\nu} H\right. \\
& \left.+\frac{1}{2} M^{2} R \Omega^{2}-\frac{\lambda}{4} \phi^{4}-\frac{\xi}{4} \eta^{4}-\frac{\omega}{4} H^{4}\right)
\end{aligned}
$$

where,

$$
\Omega^{2}=\left(1-\frac{1}{6 M^{2}} \phi^{2}-\frac{1}{6 M^{2}} H^{2}\right) .
$$

Now, the contact interactions arising from graviton exchange must be incorporated into the action [5] and these again have the effect of forcing one into the Einstein frame. This means that the disorder-order phase transition proceeds directly from pre-Plankian chaos to an Einstein frame and there is no physical meaning to the "Jordan frame." However, as in the NSI case, the form of the contact terms is that of a Weyl transformation, even though the true metric remains invariant.

To incorporate the contact terms we therefore perform a formal Weyl transformation to the Einstein frame,

$$
\begin{aligned}
S= & \int \sqrt{-g}\left(\frac{1}{2} \Omega^{-2} \partial_{\rho} \phi \partial^{\rho} \phi+\frac{1}{2} \Omega^{-2} \partial_{\rho} H \partial^{\rho} H\right. \\
& +\frac{3 M^{2}}{4} \Omega^{-4} \partial_{\rho} \Omega^{2} \partial^{\rho} \Omega^{2}+\frac{1}{2} M^{2} R \\
& \left.-\frac{\lambda}{4} \Omega^{-4} \phi^{4}-\frac{\xi}{4} \Omega^{-4} \eta^{4}-\frac{\omega}{4} \Omega^{-4} H^{4}\right)
\end{aligned}
$$

We are interested in the one loop radiative analysis of the $\mathrm{BEH}$ mass which will parallel the analysis of the scale noninvariant model. The main effects will come through the potential, $\frac{\xi}{4} \Omega^{-4} \eta^{4}$ and we presently set $\lambda=\omega=0$. The contribution of this term to the BEH mass is zero at the classical minimum of the potential, but will be generated at one loop, order $\hbar$.
Following the Coleman-Weinberg procedure [8] we shift the fields to classical background VEVs, plus small quantum corrections that are $\mathrm{O}(\sqrt{\hbar})$, and the leading one-loop result is then $\mathrm{O}(\hbar)$ ). In the following we define the parameters:

$\gamma_{i}=\left(1-\alpha_{i}\right), \quad \gamma=\frac{1}{2}\left(\gamma_{\phi}+\gamma_{H}\right), \quad \gamma^{\prime}=\frac{1}{2}\left(\gamma_{\phi}-\gamma_{H}\right)$.

From the constraint of Eq. (40) we obtain:

$$
\begin{aligned}
V_{0} & =\frac{\xi}{4} \Omega^{-4} \eta^{4} \\
& =9 \xi M^{4}\left(1-\gamma_{\phi} \frac{\phi^{2}}{6 M^{2}}-\gamma_{H} \frac{H^{2}}{6 M^{2}}\right)^{2}\left(1-\frac{\phi^{2}}{6 M^{2}}-\frac{H^{2}}{6 M^{2}}\right)^{-2} .
\end{aligned}
$$

As mentioned above, the kinetic terms of the scalars $\phi$ and $H$ are $S O(2)$ invariant, and so too the potential in the limit $\alpha_{\phi}=\alpha_{H}$, or $\gamma^{\prime}=0$. This will lead to an important constraint on our results and simplify our calculation. Since the kinetic terms are $S O(2)$ invariant we can use a polar representation of the fields, $(\phi, H) \rightarrow(\rho, \theta)$ :

$\phi^{2}+H^{2}=\rho^{2} \quad \phi=\rho \cos \theta, \quad H=\rho \sin \theta$.

As a notational convenience we set $6 M^{2}=1$ and restore this factor at the end of the calculation. Hence, $\Omega^{2}=\left(1-\phi^{2}-H^{2}\right) \rightarrow\left(1-\rho^{2}\right)$, and the classical field VEV's will be $\rho_{0}$ and $\theta_{0}$,

$\phi_{0}=\rho_{0} \cos \theta_{0} \quad$ and $\quad H_{0}=\rho_{0} \sin \theta_{0} \approx \rho_{0} \theta_{0}$.

We will be interested in the BEH boson mass term, to order $H_{0}^{2} \approx\left(\rho_{0} \theta_{0}\right)^{2}$. The "small" polar quantum fluctuations are $\mathcal{O}(\epsilon)=\sqrt{\hbar}$ :

$$
\rho=\rho_{0}+\epsilon r ; \quad \theta=\theta_{0}+\epsilon \vartheta .
$$

The polar coordinates have the advantage of diagonalizing the kinetic terms. Expanding the kinetic terms to $\mathrm{O}(\hbar)$ we see:

$$
\begin{aligned}
S_{\mathrm{KT}}= & \int \sqrt{-g}\left(\frac{1}{2} \Omega^{-2} \partial_{\rho} \phi \partial^{\rho} \phi+\frac{1}{2} \Omega^{-2} \partial_{\rho} H \partial^{\rho} H\right. \\
& \left.\left.+\frac{3 M^{2}}{4} \Omega^{-4} \partial_{\rho} \Omega^{2} \partial^{\rho} \Omega^{2}\right)\right) \\
\rightarrow & \int \sqrt{-g}\left(\frac{1}{2}\left(\Omega_{0}^{-4}\right) d r d r+\frac{1}{2} \rho_{0}^{2} \Omega_{0}^{-2}(d \vartheta d \vartheta)\right) .
\end{aligned}
$$

Then canonically normalizing the fields we have 


$$
S_{\mathrm{KT}}=\int \sqrt{-g}\left(\frac{1}{2} d \hat{r} d \hat{r}+\frac{1}{2} d \hat{\vartheta} d \hat{\vartheta}\right)
$$

where,

$$
\begin{aligned}
Z^{-1} r & =\hat{r}, \quad K^{-1} \vartheta=\hat{\vartheta}, \quad Z=\Omega_{0}^{2}, \\
K & =\Omega_{0} \rho_{0}^{-1}, \quad \Omega_{0}^{2}=\left(1-\rho_{0}^{2}\right) .
\end{aligned}
$$

Turning to the potential, we have:

$$
V_{0} \rightarrow f\left(1-\gamma_{\phi}(\rho \cos \theta)^{2}-\gamma_{H}(\rho \sin \theta)^{2}\right)^{2}\left(1-\rho^{2}\right)^{-2}
$$

where $f=\xi / 4$. We see that the $S O(2)$ symmetry is explicitly broken by nonzero $2 \gamma^{\prime}=\gamma_{\phi}-\gamma_{H}$.

We can go to the classical minimum of Eq. (53) and expand in the normalized quantum fluctuations. The classical minimum is a flat direction that corresponds to:

$$
1-\rho_{0}^{2}\left(\gamma_{\phi}+\left(\gamma_{H}-\gamma_{\phi}\right)\left(\sin ^{2} \theta_{0}\right)\right)=0
$$

Since the potential is proportional to the left-hand side (lhs) squared, the classical minimum has a vanishing energy and hence the Higgs mass term arises at $O(\hbar)$.

The renormalized mass matrix squared in the $(\hat{r}, \hat{\vartheta})$ basis is given by

$$
\Xi^{2}=f\left(\begin{array}{cc}
Z^{2} \frac{d^{2}}{d \hat{r}^{2}} V_{0} & Z K \frac{d^{2}}{d \hat{r} d \hat{\vartheta}} V_{0} \\
Z K \frac{d^{2}}{d \hat{r} d \hat{\vartheta}} V_{0} & K^{2} \frac{d^{2}}{d \hat{\vartheta}^{2}} V_{0}
\end{array}\right)
$$

With the constraint of Eq. (54) used to eliminate $\rho_{0}$, we find to $O\left(\theta_{0}^{2}\right)$ :

$$
\Xi^{2}=8 f\left(\begin{array}{cc}
\gamma+\gamma^{\prime}-2 \gamma^{\prime} \theta_{0}^{2} & -2 \gamma^{\prime} \theta_{0} X \\
-2 \gamma^{\prime} \theta_{0} X & 4 \theta_{0}^{2} \frac{\left(\gamma^{\prime}\right)^{2}}{\gamma+\gamma^{\prime}-1}
\end{array}\right)+O\left(\theta_{0}^{3}\right)
$$

where

$$
X=\left(\frac{\gamma+\gamma^{\prime}}{\gamma+\gamma^{\prime}-1}\right)^{1 / 2}
$$

The trace of $(\Xi)^{4}$ is then obtained,

$$
\begin{aligned}
\operatorname{Tr}(\Xi)^{4}= & \left(64 f^{2}\left(\gamma+\gamma^{\prime}\right)^{2}-256 f^{2}\left(\gamma+\gamma^{\prime}\right) \gamma^{\prime}\left(\gamma-\gamma^{\prime}-1\right)\right. \\
& \left.\times \frac{\theta_{0}^{2}}{\gamma+\gamma^{\prime}-1}+O\left(\theta_{0}^{4}\right)\right)
\end{aligned}
$$

and the Higgs mass term is the $O\left(\theta_{0}^{2}\right)$ term.

We restore $6 M^{2}$, and note that the normalized Higgs field in the polar representation at the potential minimum, Eq. (54), where $1-\gamma_{\phi} \rho_{0} \approx 0$, is now:

$$
h=\Omega_{0}^{-1} \rho_{0} \sin \theta_{0} \rightarrow K \theta_{0}=\frac{1}{\sqrt{\left(\gamma+\gamma^{\prime}-1\right)}} \theta_{0}
$$

Using the Coleman Weinberg form for the induced potential, Eq. (23), the BEH mass term is given by:

$\Gamma=-\frac{3}{2 \pi^{2}} M^{2} \xi^{2} \gamma^{\prime}\left(\gamma+\gamma^{\prime}\right)\left(\gamma-\gamma^{\prime}-1\right) h^{2} \ln \left(\Lambda^{2} / \mu^{2}\right)$

This vanishes in the $\gamma^{\prime}=0$ limit in which case the spontaneously broken $S O(2)$ symmetry implies that the BEH scalar is a Nambu-Goldstone boson and must then have vanishing mass. In terms of the original nonminimal couplings we have:

$$
\Gamma=-\frac{3}{4 \pi^{2}} \xi^{2}\left(1-\alpha_{\phi}\right)\left(\alpha_{H}-\alpha_{\phi}\right) \alpha_{H} M^{2} h^{2} \ln \left(\Lambda^{2} / \mu^{2}\right)
$$

\section{B. Calculation of the radiative correction with source terms}

In the calculation of the previous section we worked with the action of Eq. (42) evaluated at the classical potential minimum, Eq. (54). As a result it was not necessary to include source terms and the renormalized mass matrix, Eq. (56), involved only a a single field that we took to be $\theta$. More generally we may add sources for $H$ and $\phi$ to the action which, as discussed in Sec. II, allows us to study the effective potential as a function of both the classical fields $h$ and $\kappa$ where $H=h+\epsilon x, \chi=\kappa+\epsilon y$, unconstrained by the classical minimum condition Eq. (54).

The potential still has the form given in Eq. (46) and the unrenormalized mass squared mass matrix for the quantum fluctuations $(x, y)$ is given by:

$$
\frac{1}{2}\left(\begin{array}{ll}
y & x
\end{array}\right) \Xi_{0}^{2}\left(\begin{array}{l}
y \\
x
\end{array}\right), \quad \Xi_{0}^{2}=\left.\left(\begin{array}{cc}
\frac{\partial^{2} V_{0}}{\partial \phi^{2}} & \frac{\partial^{2} V_{0}}{\partial \phi \partial H} \\
\frac{\partial^{2} V_{0}}{\partial \phi \partial H} & \frac{\partial^{2} V_{0}}{\partial H^{2}}
\end{array}\right)\right|_{\phi=\kappa, H=h} .
$$

Without the constraint of Eq. (54) the resulting form is algebraically lengthy and requires the use of Mathematica or Maple to evaluate it, so we do not quote the result here.

Turning to the normalization of the fields, using:

$$
\frac{3 M^{2}}{4} \Omega^{-4} \partial_{\rho} \Omega^{2} \partial^{\rho} \Omega^{2}=\Omega^{-4}\left(\frac{1}{6} \phi^{2}(\partial \phi)^{2}+\frac{1}{6} H^{2}(\partial H)^{2}\right)
$$

the kinetic energy term may be rewritten as: 


$$
\begin{aligned}
K E= & \frac{3 M^{2}}{4} \Omega^{-4} \partial_{\rho} \Omega^{2} \partial^{\rho} \Omega^{2}+\frac{1}{2} \Omega^{2}\left(\partial^{\mu} \phi \partial_{\mu} \phi+\partial_{\mu} H \partial^{\mu} H\right) \\
= & \frac{1}{2} \Omega^{-4}\left(\left(1-\frac{1}{6} H^{2}\right)(\partial \phi)^{2}\right. \\
& \left.+\left(1-\frac{1}{6} \phi^{2}\right)(\partial H)^{2}+\frac{2}{6} \phi H \partial \phi \partial H\right) .
\end{aligned}
$$

Thus the kinetic energy has the form:

$$
\frac{1}{2}\left(\begin{array}{ll}
\partial y & \partial x
\end{array}\right) Z\left(\begin{array}{l}
\partial y \\
\partial x
\end{array}\right), \quad Z=\Omega(\kappa, h)^{-4}\left(\begin{array}{cc}
1-\frac{h^{2}}{6} & \frac{h \kappa}{6} \\
\frac{h \kappa}{6} & 1-\frac{\kappa^{2}}{6}
\end{array}\right) .
$$

The renormalized physical mass ${ }^{2}$ matrix is now:

$$
\Xi_{1}^{2}=Z^{-1 / 2} \Xi_{0}^{2} Z^{-1 / 2}
$$

which, inserted in Eq. (23), gives the one loop contribution to the quantum effective potential.

To compare with the previous result we can use this to calculate the $\mathrm{BEH}$ mass about the minimum of the potential which is given by $h=0$ and $\phi=\phi_{0}+\phi_{1}$, where $\phi_{0}=\sqrt{6 / \alpha_{\phi}}$ is the minimum of $V_{0}$, and $\phi_{1}$ is a correction to the minimum due to the one loop correction, $V_{1}$, given by:

$$
\phi_{1}=-\left.\frac{\partial V_{1}}{\partial \phi}\left(\frac{\partial^{2} V_{0}}{\partial \phi^{2}}\right)^{-1}\right|_{\phi=\phi_{0}}
$$

At the minimum the radiative correction to the BEH mass at one loop order is given by:

$\delta m_{h^{2}}=-\frac{1}{768 \pi^{2}} f_{0}^{4} M^{2}\left(1-\alpha_{\phi}\right)\left(\alpha_{H}-\alpha_{\phi}\right) \alpha_{H} h^{2} \ln \left(\Lambda^{2} / \mu^{2}\right)$

agreeing with the result of Eq. (61), with $f_{0}^{2}=24 \xi$, from $C=(3 / 8) M^{4} f_{0}^{2}=9 M^{4} \xi$.

We have also done a calculation in which we choose to eliminate $\phi$, rather that $\eta$, using the constraint of Eq. (40). This has the advantage of rendering the calculation similar to that of the NSI case of Sec. II. While this method does spoil the manifest $S O(2)$ symmetry, it yields the same result as Eq. (61) which displays the $S O(2)$ symmetry as $\gamma^{\prime} \rightarrow 0$. The calculation also checks whether such a change of variables affects the result-indeed we find it does not. The details are rather lengthy and we will not present them here.

\section{CONCLUSIONS}

We have provided detailed calculations of the low energy effective theory, in particular, the BEH ("Higgs") boson mass, emerging from mixed Higgs $/ R^{2}$ inflation models.
We have considered the standard nonscale-invariant form, and we have also introduced a scale invariant form where the scale symmetry is broken spontaneously inertially.

A key point here is that the contact terms show that the theory formulated in the Jordan frame involving anomalous couplings of the scalars to the Ricci scalar is identical to the Einstein frame with only an Einstein Hilbert term, $M_{P}^{2} R$, and higher dimension operators. The only ambiguity is the choice of source terms, equivalently, the order parameters of the effective potential. In the original Higgs $/ R^{2}$ model we argued the natural choice is $\chi$ and $H$, where $\chi$ is the field with a natural canonical kinetic term. In the scale invariant case the choice is any two of three of the original set of fields, $\phi, \eta$, and $H$, introduced when defining the theory. These are constrained by the kernel of the Weyl current, $K$, which develops a VEV that spontaneously breaks Weylscale invariance.

Once the source terms are specified, the radiative corrections are unambiguous. Our results show that in both case the radiative corrections to the BEH mass do not vanish in the "conformally coupled" limit, $\alpha_{H}=1$, in contrast to the results obtained by previous authors [18].

A renormalization group approach, introduced here, significantly improves our understanding of these theories and expedites these analyses. We have only touched upon the RG approach here in application to the nonscale invariant Higgs $/ R^{2}$ model. In the RG approach the choice of variables is embedded in the choice of boundary conditions on the RG equations. This will be developed elsewhere in greater detail [10]. Note that the running of the Higgs boson mass is not due to the running of the $\alpha_{i}$ but rather mainly due to the effects of other relevant operators that emerge from the nonpolynomial Starobinsky potential at the Planck-scale. At the single-log order discussed here, the $\alpha_{H}$ enters in the UV only as a boundary condition upon the relevant couplings.

The determination of the radiative corrections to the BEH mass is of importance to the viability of the Starobinsky inflationary model. To generate an acceptable period of inflation the model requires a very heavy scaleron, of $O\left(10^{13}\right) \mathrm{GeV}$, and the coupling of the scaleron to the BEH scalar typically gives an unacceptably large contribution to its mass leading to a severe hierarchy problem. Conceivably, the $S O(2)$ symmetry of the scale invariant model, or its generalization, might allow the possibility of a protected BEH scalar mass, as a pseudo-Nambu-Goldstone boson. We will discuss the implications of our results for the hierarchy problem in detail elsewhere [35].

\section{ACKNOWLEDGMENTS}

We thank P. Ferreira and J. Noller for discussions. This manuscript has been authored in part by Fermi Research Alliance, LLC under Contract No. DE-AC02-07CH11359 with the U.S. Department of Energy, Office of Science, Office of High Energy Physics. 
Note added.-After completion of this work we became aware of the interesting papers of C. Steinwachs et al. which address the loop level consistency issues in these models [7]. We believe that the gravitational contact terms resolve many of the issues encountered by these authors. Moreover, the Wilson-style renormalization group approach exhibited in Sec. II D provides a simpler calculational framework and will be developed further elsewhere.

\section{APPENDIX A: REGULARIZED LOOP INTEGRALS AND QUANTUM SCALE BREAKING}

We are interested in loop induced effective potentials. These are contained in the log of the path integral: $\Gamma=i \ln P$. In the case of a real scalar field of physical mass, $m$, we have:

$$
P=\prod_{k}\left(k^{2}-m^{2}\right)^{-1 / 2}=\operatorname{det}\left(k^{2}-m^{2}\right)^{-1 / 2}
$$

hence,

$$
\Gamma=i \ln P=-\frac{i}{2} \int \frac{d^{4} k}{(2 \pi)^{4}} \ln \left(k^{2}-m^{2}+i \epsilon\right)
$$

This can be evaluated with a Wick rotation and Euclidean momentum space cutoff:

$$
\begin{aligned}
\Gamma= & \frac{1}{2} \int_{0}^{\Lambda} \frac{d^{4} k_{E}}{(2 \pi)^{4}} \ln \left(\frac{k_{E}^{2}+m^{2}}{\Lambda^{2}}\right)+(\text { irrelevant constants }) \\
= & \frac{1}{64 \pi^{2}}\left(\ln \frac{\Lambda^{2}+m^{2}}{\Lambda^{2}} \Lambda^{4}-m^{4} \ln \frac{\Lambda^{2}+m^{2}}{\Lambda^{2}}-\frac{1}{2} \Lambda^{4}\right. \\
& \left.+\Lambda^{2} m^{2}+m^{4} \ln \frac{m^{2}}{\Lambda^{2}}\right)
\end{aligned}
$$

The cutoff can be viewed is a spurious parameter, introduced to make the integral finite and not part of the defining action. The only physically meaningful dependence upon $\Lambda$ is contained in the logarithm, where it reflects scale symmetry breaking by the quantum trace anomaly. Powers of $\Lambda$, e.g., $\Lambda^{4}, \Lambda^{2} m^{2}$. spuriously break scale symmetry and are not part of the classical action [36].

It is therefore conceptually useful to have a definition of the loops in which the spurious powers of $\Lambda$ do not arise. This can be done by defining the loops applying projection operators on the integrals. The projection operator

$$
P_{n}=\left(1-\frac{\Lambda}{n} \frac{\partial}{\partial \Lambda}\right)
$$

removes any terms proportional to $\Lambda^{n}$. Since the defining classical Lagrangian has mass dimension 4 and involves no terms with $\Lambda^{2} m^{2}$ or $\Lambda^{4}$, we define the regularized loop integrals as:

$$
\begin{aligned}
\Gamma \rightarrow & \frac{1}{2} P_{2} P_{4} \int_{0}^{\Lambda} \frac{d^{4} k_{E}}{(2 \pi)^{4}} \ln \left(\frac{k_{E}^{2}+m^{2}}{\Lambda^{2}}\right) \\
& +(\text { irrelevant constants }) \\
= & -\frac{1}{64 \pi^{2}}\left(m^{4}\left(\ln \frac{\Lambda^{2}}{m^{2}}-1\right)+O\left(\frac{m^{6}}{\Lambda^{2}}\right)\right)
\end{aligned}
$$

where we take the limit $\Lambda \gg m$ to suppress $O\left(m^{6} / \Lambda^{2}\right)$ terms. It appears this can be consistently used in $N$-loop calculations with $\left(P_{2} P_{4}\right)^{N}$ though we now apply it only to single loop amplitudes.

Equation (A5) defines the quantum effective potential for a classical real scalar field with mass term $V_{c}=m^{2} \phi^{2} / 2$ :

$$
\Gamma=V_{c}-\frac{1}{64 \pi^{2}} m^{4} \ln \frac{M^{2}}{m^{2}}
$$

where we have replaced the cut-off by the Planck-scale.

As a check we can compute the $\beta$-function for a quartic coupling constant. Consider $V_{c}=\lambda \phi^{4} / 4$. We expand $\phi$ in a classical plus quantum fluctuation field $x, \phi \rightarrow \phi_{c}+\epsilon x$ and $V_{c} \rightarrow \lambda \phi_{c}^{4} / 4+3 \hbar \lambda \phi_{c}^{2} x^{2} / 2$, where terms linear in $x$ integrate to zero. We see that the physical mass of $x$ is $m^{2}=3 \lambda \phi_{c}^{2}$ and using $\Gamma$ we have:

$$
\Gamma=\frac{\lambda}{4} \phi_{c}^{4}-\frac{9 \lambda^{2}}{64 \pi^{2}} \phi_{c}^{4} \ln \frac{M^{2}}{m^{2}}
$$

Therefore, we see that $\lambda$ runs as:

$$
\lambda(\mu)=\lambda_{0}-\frac{9 \lambda^{2}}{8 \pi^{2}} \phi^{4} \ln \frac{M}{\mu}
$$

where the initial value at the Planck-scale is $\lambda_{0}$ and the $\beta$-function is

$$
\beta=\frac{\partial \lambda(\mu)}{\partial \ln (\mu)}=\frac{9 \lambda^{2}}{8 \pi^{2}}
$$

a well-known result (see Refs. [8,9] and references therein).

The above result is $\mathcal{O}(\hbar)$. since the expansion in $\hbar$ is an expansion in the number of loops. Note that $\Gamma=i \hbar \ln (i S / \hbar)$, so a classical action $S$ produces an $\mathrm{O}$ (1) result in the $\hbar$ expansion. The quantum field kinetic terms are $\sim S \sim \int \hbar(\partial \phi)^{2}$ hence a propagator is $1 / \hbar$ and a single Feynman loop is $\Gamma \propto \hbar \ln \left(i \int \hbar(\partial \phi)^{2} / \hbar\right) \sim \hbar ; N$ loops are $\propto \hbar^{N}$.

\section{APPENDIX B: EQUIVALENCE OF ON-SHELL FIELD CONFIGURATIONS AND AMBIGUITIES IN EFFECTIVE POTENTIALS}

It is fairly easy to give a formal proof of the equivalence of different field choices for calculation of effective potentials with on-shell classical background fields. 
Our proof for $N=1$ fields is schematic and readily can be generalized to $N$ fields. Consider the action:

$$
S=\int \frac{1}{2} \partial \phi \partial \phi-V(\phi)
$$

For an effective potential, such as a Coleman-Weinberg calculation, $V(\phi)$ is understood to contain the source terms. Note that we expand in a classical background field $\phi_{0}$ plus a quantum fluctuation, $x, \phi=\phi_{0}+\epsilon x$ (where $\epsilon=\sqrt{\hbar}$ ), and we have,

$$
\begin{gathered}
\int \frac{1}{2} \partial \phi_{0} \partial \phi_{0}+\partial \phi_{0} \partial x+\frac{1}{2} \partial x \partial x-V\left(\phi_{0}\right) \\
-\frac{d}{d \phi_{0}} V\left(\phi_{0}\right) x-\frac{1}{2} \frac{d^{2}}{d \phi_{0}^{2}} V\left(\phi_{0}\right) x^{2}
\end{gathered}
$$

We now make the assumption that $\phi_{0}$ is "on-shell," i.e., satisfies its classical equation of motion:

$$
\partial^{2} \phi_{0}=-\frac{d}{d \phi_{0}} V\left(\phi_{0}\right)
$$

hence, integrating by parts,

$S=\int \frac{1}{2} \partial \phi_{0} \partial \phi_{0}+\frac{1}{2} \partial x \partial x-V\left(\phi_{0}\right)-\frac{1}{2}\left(\frac{d^{2}}{d \phi_{0}^{2}} V\left(\phi_{0}\right)\right) x^{2}$

If we are only interested in a potential, we simplify by assuming $\phi_{0}=$ spatially (and temporally) constant. Therefore the "on-shell" condition requires:

$$
\frac{d}{d \phi_{0}} V\left(\phi_{0}\right)=0
$$

i.e., $\phi_{0}$ must be at the minimum of the potential. Bear in mind that the source currents shift the true potential minimum to an arbitrary value, $\phi_{0}$, and the potential we compute will correspond to the energy of a state with lowest energy subject the constraint that the expectation value of $\phi$ is $\phi_{0}$.

Now suppose $\phi(\chi)$ is a function of a new field $\chi$. Then

$$
S=\int \frac{1}{2} \partial \phi \partial \phi-V(\phi) \rightarrow \int \frac{1}{2}\left(\frac{\partial \phi}{\partial \chi}\right)^{2} \partial \chi \partial \chi-V(\phi(\chi))
$$

and now we want the expansion $\chi=\chi_{0}+\epsilon y$ with spatially constant $\chi_{0}$

$$
\begin{aligned}
S= & \int \frac{1}{2} \partial \phi \partial \phi-V(\phi) \\
& \rightarrow \int \frac{1}{2}\left(\frac{\partial \phi_{0}}{\partial \chi_{0}}\right)^{2} \partial y \partial y-V\left(\phi_{0}\right)-\frac{\partial \phi_{0}}{\partial \chi_{0}} \frac{d}{d \phi_{0}} V\left(\phi_{0}\right) y \\
& -\frac{1}{2}\left(\frac{\partial \phi_{0}}{\partial \chi_{0}} \frac{d}{d \phi_{0}}\left(\frac{\partial \phi_{0}}{\partial \chi_{0}} \frac{d}{d \phi_{0}}\right) V\left(\phi_{0}\right)\right) y^{2} .
\end{aligned}
$$

Examine the last term, which takes the form

$$
\begin{aligned}
- & \frac{1}{2}\left(\frac{\partial \phi_{0}}{\partial \chi_{0}}\right)^{2}\left(\frac{d^{2}}{d \phi_{0}^{2}}\left(V\left(\phi_{0}\right)\right)\right) x^{2} \\
& -\frac{1}{2} \frac{\partial^{2} \phi_{0}}{\partial \chi_{0}^{2}}\left(\frac{\partial \phi_{0}}{\partial \chi_{0}} \frac{\partial}{\partial \phi_{0}} V\left(\phi_{0}\right)\right) x^{2} .
\end{aligned}
$$

The last term is problematic off-shell, but on-shell we have:

$$
\frac{\partial \phi_{0}}{\partial \chi_{0}} \frac{\partial}{\partial \phi_{0}}\left(V\left(\phi_{0}\left(\chi_{0}\right)\right)\right)=\frac{\partial}{\partial \chi_{0}} \hat{V}\left(\chi_{0}\right)=0
$$

That is, $\chi_{0}$ must be a minimum of the new potential:

$$
\hat{V}\left(\chi_{0}\right)=V\left(\phi_{0}\left(\chi_{0}\right)\right)
$$

which corresponds to the minimum of $V\left(\phi_{0}\right)$. Hence the action is

$$
\begin{aligned}
S & \rightarrow \int\left(\frac{1}{2}\left(\frac{\partial \phi_{0}}{\partial \chi_{0}}\right)^{2} \partial y \partial y-\hat{V}\left(\chi_{0}\right)\right. \\
& \left.-\frac{1}{2}\left(\frac{\partial \phi_{0}}{\partial \chi_{0}}\right)^{2} \frac{d^{2}}{d \phi_{0}^{2}} V\left(\phi_{0}\right) y^{2}\right)
\end{aligned}
$$

Renormalizing $y$ yields:

$$
\int\left(\frac{1}{2} \partial y \partial y-\hat{V}\left(\chi_{0}\right)-\frac{1}{2} \frac{d^{2}}{d \phi_{0}^{2}} V\left(\phi_{0}\right) y^{2}\right) .
$$

Since

$$
\frac{1}{2} \frac{d^{2}}{d \phi_{0}^{2}} V\left(\phi_{0}\right)=m^{2}
$$

is common to both the $\chi$ and $\phi$ theories, therefore the quantum potentials, $\propto \int \ln \left(k^{2}+m^{2}\right)$ must be equivalent when the background fields are localized at the minimum of the potential (including source terms).

We can test this theorem in a simplified model (we will use the model in Appendix $\mathrm{C}$ to illustrate the ambiguities that result if there are different Weyl frames). We consider a model which is similar to those encountered in Starobinsky inflation. It has the fields $\Omega, H$, and external sources $(J, K)$ with action: 


$$
\begin{aligned}
S= & \int \frac{1}{2} \partial H \partial H+\frac{1}{2} M^{2} \partial \ln \Omega \partial \ln \Omega-V(H, \Omega) \\
& -J M \ln (\Omega)-K H
\end{aligned}
$$

where

$$
V(H, \Omega)=\frac{1}{4} M^{4}\left(\Omega^{2}-\left(1+\frac{\alpha}{M^{2}} H^{2}\right)\right)^{2}
$$

We use the representation of the field, $\Omega(\chi)=\exp (\chi / M)$, and define classical background fields and quantum fluctuations $\sqrt{\hbar}=\epsilon, H=h+\epsilon x$, and $\chi=\kappa+\epsilon y$.

Our procedure is as follows:

(1) Expand the action to $\mathrm{O}(\hbar)$ including sources,

$$
V(H, \Omega)-J M \ln (\Omega)-K H \rightarrow W(J, K ; h, \kappa, \epsilon x, \epsilon y) .
$$

(2) Determine on-shell conditions for $(J, K)$ from the $O\left(\epsilon^{0}\right)$ (classical term) of the potential by imposing the minimum conditions on $(h, \kappa)$.

$$
\begin{aligned}
\frac{d}{d \kappa} W(J, K ; h, \kappa, 0,0) & =0 \\
\frac{d}{d h} W(J, K ; h, \kappa, 0,0) & =0 .
\end{aligned}
$$

This determines $J, K$ as functions of $(h, \kappa)$. Legendre transformation
(3) Define the effective potential by performing the

$$
\begin{aligned}
& W(J, K ; h, \kappa, \epsilon x, \epsilon y)-\frac{d W}{d J} \kappa-\frac{d W}{d K} h \\
& =\Gamma(\kappa, h,, \epsilon x, \epsilon y) .
\end{aligned}
$$

At this stage the terms linear in $\epsilon x, \epsilon y$ cancel. Hence the functions $J, K$ completely drop out if the source terms are linear in $x, y$. Otherwise, $J, K$ will enter the quadratic and higher terms.

(4) Expand $\Gamma(\kappa, h,, \epsilon x, \epsilon y)$ to determine the mass $^{2}$ matrix of the quantum fluctuations $\epsilon x, \epsilon y$ at order $\epsilon^{2}$

$\Gamma(\kappa, h,, \epsilon x, \epsilon y)=\frac{1}{2}\left(\begin{array}{l}y \\ x\end{array}\right)^{T} \Xi^{2}(\kappa, h)\left(\begin{array}{l}y \\ x\end{array}\right)$

and the kinetic terms and renormalization constant matrix

$$
\frac{1}{2}\left(\begin{array}{c}
\partial y \\
\partial x
\end{array}\right)^{T} Z(\kappa, h)\left(\begin{array}{l}
\partial y \\
\partial x
\end{array}\right) .
$$

(5) Integrating out $(\epsilon x, \epsilon y)$ the quantum effective potential is now determined,

$$
-\frac{1}{64 \pi^{2}} \operatorname{Tr}\left(\left(Z \Xi^{2}(\kappa, h)\right)^{2}\right) \ln \left(\Lambda^{2} / m^{2}\right) .
$$

We apply this to two examples with different quantum field definitions.

Example (1): With $\Omega=\exp (\chi / M)$, the potential $V(H, \Omega)$ in Eq. (B15), and source terms $-J M \ln (\Omega)-K H$, and we follow the procedure, find that $J, K$ cancel in the mass matrix

$$
\begin{aligned}
\Xi^{2} & =\frac{1}{2}\left(\begin{array}{cc}
6 \kappa M-\alpha h^{2}+6 \kappa^{2}+M^{2} & -\alpha h M-2 \alpha h \kappa \\
-\alpha h M-2 \alpha h \kappa & -\alpha \kappa M+\frac{3}{2} \alpha^{2} h^{2}-\alpha \kappa^{2}
\end{array}\right)\left(\begin{array}{l}
y \\
x
\end{array}\right) \\
Z^{-1} & =\left(\begin{array}{ll}
1 & 0 \\
0 & 1
\end{array}\right)\left(\begin{array}{l}
y \\
x
\end{array}\right)
\end{aligned}
$$

Keeping only quadratic order in $\kappa, h$ we find:

$$
\operatorname{Tr}\left(Z^{-1} \Xi^{2}\right)^{2}=\left(\frac{1}{4} M^{4}+3 M^{3} \kappa\right)+\left(\frac{1}{2} \alpha^{2} M^{2}-\frac{1}{2} M^{2} \alpha\right) h^{2}+O\left(h^{4}, \kappa^{2}, \kappa h^{2}\right)
$$

Example (2) Now consider the alternative parametrization $(\tilde{\chi}, H)$ where $\chi=M \ln (1+\tilde{\chi} / K)$. We keep the same sources $-J M \ln (\Omega)$ and $V-K H$. We now need to expand the log, and we find that $J$ appears in quadratic terms of $(x, y)$ due to nonlinear term $J \tilde{\chi}^{2}$ :

$$
\begin{aligned}
\Xi^{2} & =\frac{1}{2}\left(\begin{array}{cc}
4 \kappa M-\alpha h^{2}+4 \kappa^{2}+M^{2}-\frac{1}{M} \kappa \alpha h^{2}-\frac{1}{M^{2}} \kappa^{2} \alpha h^{2} & -\alpha h \kappa-\alpha h M \\
-\alpha h \kappa-\alpha h M & -\alpha \kappa M+\frac{3}{2} \alpha^{2} h^{2}-\frac{1}{2} \alpha \kappa^{2}
\end{array}\right)\left(\begin{array}{l}
y \\
x
\end{array}\right) \\
Z^{-1} & =\left(\begin{array}{cc}
\left(1+\frac{2}{M} \kappa\right) & 0 \\
0 & 1
\end{array}\right)
\end{aligned}
$$


we obtain $\operatorname{Tr}\left(Z^{-1} \Xi^{2}\right)^{2}=\left(\frac{1}{4} M^{4}+3 M^{3} \kappa\right)+\left(\frac{1}{2} \alpha^{2} M^{2}-\right.$ $\left.\frac{1}{2} M^{2} \alpha\right) h^{2}+O\left(h^{4}, \kappa^{2}, \kappa h^{2}\right)$ These expressions agree and yield the same potential though the intermediate steps are quite different. The equivalence is a consequence of the above theorem.

\section{APPENDIX C: SOURCE AMBIGUITIES}

The equivalence proved in Appendix B requires that the same sources should be used in both parametrizations. Thus in Example (2) above it was important to choose the same source, $-J M \ln (\Omega) \equiv-J \chi$, an in Example (1), and the result is independent of the integration over quantum fluctuations. However, if instead we used the source $-J \tilde{\chi}$ we have changed the order parameter and the two calculations will differ. Such an ambiguity is inherent to the theory and requires the choice of "reasonable" sources when defining the theory.

To illustrate this we note that in many studies of Higgs $/ R^{2}$ inflation the anomalous coupling to the $\mathrm{BEH}$ field is not included when Weyl transforming to a pseudoEinstein frame. This residual anomalous coupling can be eliminated by a second Weyl transformation that completes the definition of the model in the Einstein frame. Weyl transformations satisfy the group property and the two Weyl transformations are equivalent to the single Weyl transformation going directly to the Einstein frame. However the fundamental variables suggested by this approach differ from that of the previous section.

The two stage model starts with rewriting the action of Eq. (2) in the form ${ }^{4}$

$$
\begin{aligned}
S= & \int d^{4} x \sqrt{-\tilde{g}}\left(\frac{M}{2} \Omega_{1}^{-2} R(g)-\frac{\xi}{4} \eta^{4}+\frac{1}{2} \partial_{\mu} H \partial^{\mu} H\right. \\
& \left.-\frac{1}{12} H^{2} R(g)-V(H)\right)
\end{aligned}
$$

where,

$$
\Omega_{1}^{-2}=1-\frac{\alpha_{\eta}}{6}\left(\frac{\eta}{M}\right)^{2}-\frac{\alpha_{H}-1}{6}\left(\frac{H}{M}\right)^{2} \equiv \exp \left(\sqrt{\frac{2}{3}} \frac{\chi}{M}\right) .
$$

We perform the first Weyl transformation:

$$
\tilde{g}=\Omega_{1}^{2} g_{1}
$$

giving,

$$
\begin{aligned}
S= & \int d^{4} x \sqrt{-g_{1}}\left(\frac{M}{2} R\left(g_{1}\right)+\frac{1}{2} \partial_{\mu} \chi \partial^{\mu} \chi+\frac{1}{2} \partial_{\mu} \tilde{H} \partial^{\mu} \tilde{H}\right. \\
& \left.-\frac{1}{12} \tilde{H}^{2} R\left(g_{1}\right)-\frac{\xi}{4} \Omega_{1}^{4} \eta^{4}-\Omega_{1}^{4} V(H)\right),
\end{aligned}
$$

in terms of the conformally rescaled field,

$$
H_{1}=\Omega_{1} H \text {. }
$$

The second Weyl transformation eliminates the residual anomalous coupling of the Higgs field and is given by,

$$
g_{1}=\Omega_{2}^{2} g_{2},
$$

with the conformal factor,

$$
\Omega_{2}^{-2}=1-\frac{1}{6}\left(\frac{H_{1}}{M}\right)^{2} \equiv \exp \left(\sqrt{\frac{2}{3}} \frac{\rho}{M}\right),
$$

giving:

$$
\begin{aligned}
S & =\int d^{4} x \sqrt{-g_{2}}\left(\frac{M}{2} R\left(g_{2}\right)+\frac{1}{2} \Omega_{2}^{2} \partial_{\mu} \chi \partial^{\mu} \chi+\frac{1}{2} \partial_{\mu} \rho \partial^{\mu} \rho+\frac{1}{2} \Omega_{2}^{2} \partial_{\mu} H_{1} \partial^{\mu} H_{1}-\frac{\xi}{4} \Omega_{2}^{4} \Omega_{1}^{4} \eta^{4}-\Omega_{2}^{4} \Omega_{1}^{4} V(H)\right) \\
& =\int d^{4} x \sqrt{-g}\left(\frac{M}{2} R(g)+\frac{1}{2} \Omega_{2}^{2} \partial_{\mu} \chi \partial^{\mu} \chi+\frac{1}{2} \Omega_{2}^{4} \partial_{\mu} H_{1} \partial^{\mu} H_{1}-\frac{\xi}{4} \Omega^{4} \eta^{4}-\Omega^{4} V(H)\right) .
\end{aligned}
$$

Since the Weyl transformations form a group, comparing to the case studied in Sec. II A we have $g_{2}=g$ and $\Omega=\Omega_{2} \Omega_{1}$. Comparing with Eq. (2) we see that the difference is that now the natural choice of fundamental

\footnotetext{
${ }^{4}$ Here, to keep the algebraic complexity to a minimum while demonstrating the ambiguity, we do include a component of the $\mathrm{BEH}$ scalar in the first Weyl transformation. This component vanishes in the conformal limit.
}

variables is $\chi$ and $\tilde{H}$. Proceeding as in Sec. II we obtain,

$\Gamma=\frac{1}{768 \pi^{2}} M^{2} f_{0}^{4}\left(\left(\alpha_{H}-1\right)\left(\alpha_{H}-2\right)+1\right) h^{2}\left(\ln \left(\Lambda^{2} / m^{2}\right)\right)$.

A comparison with Eq. (26) shows that the mass terms differ, demonstrating the ambiguity associated with the choice of fundamental fields. 
The resolution of this ambiguity follows from the existence of the contact terms. As stressed in [5], when the BEH scalar is included, this approach implicitly requires the inclusion of contact terms to take account of its anomalous coupling to the Ricci scalar. Including this term automatically takes one to the Einstein frame so there is no meaning to the two stage Weyl transformations just discussed and there is no corresponding ambiguity.
[1] A. A. Starobinsky, Phys. Lett. 91B, 99 (1980); Adv. Ser. Astrophys. Cosmol. 3, 130 (1987).

[2] F. Englert and R. Brout, Phys. Rev. Lett. 13, 321 (1964).

[3] P. W. Higgs, Phys. Lett. 12, 132 (1964).

[4] P. W. Higgs, Phys. Rev. Lett. 13, 508 (1964).

[5] C. T. Hill and G. G. Ross, Phys. Rev. D 102, 125014 (2020).

[6] M. J. Duff, Inconsistency of quantum field theory in curved space-time, ICTP/79-80/38.

[7] A. Gundhi and C. F. Steinwachs, Eur. Phys. J. C 81, 460 (2021); A. Y. Kamenshchik and C. F. Steinwachs, Phys. Rev. D 91, 084033 (2015); M. S. Ruf and C. F. Steinwachs, Phys. Rev. D 97, 044050 (2018); 97, 044049 (2018); C. F. Steinwachs and A. Y. Kamenshchik, Phys. Rev. D 84, 024026 (2011); AIP Conf. Proc. 1514, 161 (2013).

[8] S. R. Coleman and E. J. Weinberg, Phys. Rev. D 7, 1888 (1973).

[9] C. T. Hill, Phys. Rev. D 89, 073003 (2014).

[10] C. T. Hill and G. G. Ross (to be published).

[11] C. Brans and R. H. Dicke, Phys. Rev. 124, 925 (1961).

[12] M. Shaposhnikov and D. Zenhausern, Phys. Lett. B 671, 162 (2009).

[13] M. Shaposhnikov and D. Zenhausern, Phys. Lett. B 671, 187 (2009).

[14] D. Blas, M. Shaposhnikov, and D. Zenhausern, Phys. Rev. D 84, 044001 (2011).

[15] J. Garcia-Bellido, J. Rubio, M. Shaposhnikov, and D. Zenhausern, Phys. Rev. D 84, 123504 (2011).

[16] P. G. Ferreira, C. T. Hill, and G. G. Ross, Phys. Lett. B 763, 174 (2016).

[17] P. G. Ferreira, C. T. Hill, and G. G. Ross, Phys. Rev. D 95, 043507 (2017).

[18] A. Salvio and A. Strumia, Eur. Phys. J. C 78, 124 (2018).
[19] Y.C. Wang and T. Wang, Phys. Rev. D 96, 123506 (2017).

[20] Y. Ema, Phys. Lett. B 770, 403 (2017).

[21] M. He, A. A. Starobinsky, and J. Yokoyama, J. Cosmol. Astropart. Phys. 05 (2018) 064.

[22] A. Gundhi and C. F. Steinwachs, Nucl. Phys. B954, 114989 (2020).

[23] V. M. Enckell, K. Enqvist, S. Rasanen, and L. P. Wahlman, J. Cosmol. Astropart. Phys. 01 (2020) 041.

[24] M. He, R. Jinno, K. Kamada, A. A. Starobinsky, and J. Yokoyama, J. Cosmol. Astropart. Phys. 01 (2021) 066.

[25] D. M. Ghilencea, Phys. Rev. D 98, 103524 (2018).

[26] D. M. Ghilencea, J. High Energy Phys. 03 (2019) 049.

[27] P. G. Ferreira, C. T. Hill, and G. G. Ross, Phys. Rev. D 98, 116012 (2018).

[28] M. Herranen, T. Markkanen, S. Nurmi, and A. Rajantie, Phys. Rev. Lett. 113, 211102 (2014).

[29] M. Herranen, T. Markkanen, S. Nurmi, and A. Rajantie, Phys. Rev. Lett. 115, 241301 (2015).

[30] B. Whitt, Phys. Lett. 145B, 176 (1984).

[31] A. Hindawi, B. A. Ovrut, and D. Waldram, Phys. Rev. D 53, 5583 (1996).

[32] P. G. Ferreira, C. T. Hill, J. Noller, and G. G. Ross, Phys. Rev. D 100, 123516 (2019).

[33] D. Ghilencea, J. High Energy Phys. 10 (2019) 209.

[34] P. G. Ferreira, C. T. Hill, and G. G. Ross, Phys. Rev. D 95, 064038 (2017).

[35] Starobinsky inflation and the hierarchy problem, P. T. Ferreira, C. T. Hill, J. Noller, and G. G. Ross (to be published).

[36] W. A. Bardeen, On naturalness in the standard model, FERMILAB-CONF-95-391-T. 